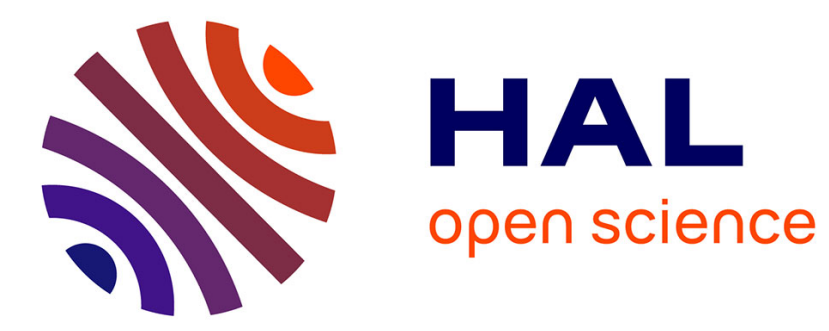

\title{
Crack opening displacements under remote stress gradient: Derivation with a canonical basis of sixth order tensors
}

\author{
Vincent Monchiet, Guy Bonnet
}

\section{- To cite this version:}

Vincent Monchiet, Guy Bonnet. Crack opening displacements under remote stress gradient: Derivation with a canonical basis of sixth order tensors. International Journal of Engineering Science, 2015, 91, pp.1-11. 10.1016/j.ijengsci.2015.01.002 . hal-01165843v2

\section{HAL Id: hal-01165843 \\ https://hal.science/hal-01165843v2}

Submitted on 18 Mar 2021

HAL is a multi-disciplinary open access archive for the deposit and dissemination of scientific research documents, whether they are published or not. The documents may come from teaching and research institutions in France or abroad, or from public or private research centers.
L'archive ouverte pluridisciplinaire HAL, est destinée au dépôt et à la diffusion de documents scientifiques de niveau recherche, publiés ou non, émanant des établissements d'enseignement et de recherche français ou étrangers, des laboratoires publics ou privés. 


\title{
Crack opening displacements under remote stress gradient: derivation with a canonical basis of sixth order tensors
}

\author{
Vincent Monchiet, Guy Bonnet \\ Université Paris-Est, Laboratoire Modélisation et Simulation Multi Echelle, \\ LMSME UMR8208 CNRS, 5 boulevard Descartes, 77454 Marne la Vallée Cedex, \\ France
}

\begin{abstract}
In this paper, we derive the crack opening displacement of a penny-shaped crack embedded in an infinite isotropic elastic medium and subjected to a remote constant stress gradient. The solution is derived by taking advantage of the solution of the equivalent ellipsoidal inclusion problem subjected to a linear polarization. The case of the penny-shaped crack is thereafter investigated by considering the case of a spheroidal cavity which has one principal axis infinitesimally small compared to both others. The derivation of the explicit solution for the inhomogeneity subjected to a remote stress gradient raises the problem of the inversion of a sixth order tensor. For the problem having a symmetry axis (this including the case of penny shaped crack), this problem can be tackled by using a decomposition on the canonical basis for transversely isotropic sixth order tensors.
\end{abstract}

Key words: Cracks, Sixth-order tensor, Anisotropy, Inhomogeneity problem

\section{Introduction}

This paper provides the explicit solution for the penny shaped crack opening displacement subjected to remote stress gradient. The problem of a crack in an infinite elastic body has been studied for a long time; there is an abondant literature on this subject which has been summarized in the classic books of $[16,17,8]$. Classically, the problem of crack submitted to an applied remote

Email addresses: vincent.monchiet@univ-paris-est.fr, tel: (33)0160957793, fax: (33)0160957795 (Vincent Monchiet, Guy Bonnet). 
strain (or equivalently stress) can be addressed using the Eshelby $[3,4]$ formalism. Eshelby's solution for inclusions and for equivalent inhomogeneity problems are fundamental to many problems in material science, mechanics of composite, etc. In the terminology of Eshelby [3] and Mura [16], an inclusion denotes a subdomain subjected to an eigenstrain while an inhomogeneity is a subdomain whose elastic properties differ from those of the surrounding medium. The main Eshelby's result is well known for the case of a prescribed constant eigenstrain: it shows that a constant strain field is generated inside an ellipsoidal inclusion [3] while the exterior point solution (outside the inclusion) is heterogeneous [4]. The Eshelby's equivalent method handles the problem of a single ellipsoidal inhomogeneity by replacing it with an inclusion having properly chosen eigenstrains. The results for the penny shaped cracks are recovered when two semi-axes of the ellipsoidal inhomogeneity are equal and the last is infinitesimally small compared to the others.

Due to its simplicity, the Eshelby's solution is the basis of numerous ways to understand the behaviour of heteregeneous and cracked materials, and finally was used extensively to construct numerous constitutive equations of these materials. A few recent references show that this field of research is still active for studying cracked materials $[11,9]$. However, some works have shown also that taking into account the influence of strain (or stress) gradients is of fundamental significance when studying cracked materials, leading to the use of constitutive equations of gradient elasticity type [15,7]. So, extending the Eshelby's formalism to inclusions and cracks submitted to remote strain (or stress) gradients allows to provide a new fundamental background to the understanding of heterogeneous materials. Such a solution was provided in [12] for the case of an ellipsoidal inclusion submitted to a remote gradient, based on older fundamental results. Indeed, following Eshelby's work, Sendeckyj [19], Moschovidis [13], Asaro and Barnett [1], generalize Eshelby's solution to the case of prescribed polynomial fields. In these studies, the following result has been proved: the strain in an ellipsoidal subdomain of an infinite linear elastic medium which undergoes an eigenstrain on the form of a polynomial of degree $N$, is also a polynomial with the same degree $N$. The expansion of the eigenstrain and the interior point solution for the strain field along polynomial functions introduce tensors of order 2, 3, 4 etc and higher order Eshelby tensors of order 6,8 etc. It has been pointed out in [16] that the strain disturbance due to a polynomial type remote strain field of degree $N$ can be simulated by an appropriate polynomial eigenstrain field of degree $N$. If the inclusion problem can be traced back to these works, the related heterogeneity problem requires the inversion of high order tensors. For example, for a remote strain which is linear with coordinates, the problem involves the inversion of a sixth order tensor. Although this inversion can be performed numerically, it is obviously of interest to derive closed form solutions which can cover many applications, as performed in [12].

In the present paper, we use a canonical basis of transversely isotropic sixth order tensors [12] which has the advantage to reduce drastically the size of the 
system for the case of a spheroidal inhomogeneity (ellipsoidal inhomogeneity with symmetry axis). It allows to provide an easy inversion of high order tensors and therefore to find an easy way to solve the heterogeneity problem. Thus, the linear system which is initially of dimension 18 reduces to five independent systems of dimension 1, 1, 2, 3 and 4 . When the case of a void is considered the problem exhibits some impotent strains and degenerates into five independent systems of dimension 1, 1, 2, 2 and 3. Finally, the case of a penny shaped crack is considered by expanding the solution with respect to the aspect ratio of the spheroidal inhomogeneity.

\section{Inhomogeneity problem with applied remote strain or stress gra- dient}

Consider an ellipsoidal inhomogeneity embedded in an infinite elastic matrix and centered at the origin of the cartesian frame $\left(x_{1}, x_{2}, x_{3}\right)$. Let us denote by $a_{1}, a_{2}, a_{3}$ the semi-axes of the ellipsoid along the three axes of the cartesian frame. The subdomain $\Omega$ of the inhomogeneity is defined by:

$$
U(x)=\eta_{i j} x_{i} x_{j}-1 \leq 0
$$

with:

$$
\eta_{i j}= \begin{cases}0 & \text { if } i \neq j \\ 1 / a_{i}^{2} & \text { if } i=j\end{cases}
$$

Both the inhomogeneity and the infinite domain are assumed to be isotropic and we denote by $\mu, \lambda$ and $\mu_{0}, \lambda_{0}$ their respective Lamé coefficients. The inhomogeneity is subjected to a remote strain field taken on the form $\varepsilon_{i j}(x)=$ $\varepsilon_{i j k}^{\infty} x_{k}$ or equivalently to the remote stress field $\sigma_{i j}^{\infty}(x)=\sigma_{i j k}^{\infty} x_{k}$ where $\varepsilon_{i j k}^{\infty}$ and $\sigma_{i j k}^{\infty}$ are constant ( $\underline{x}$-independent). This problem is called "second order heterogeneity problem" since a uniform gradient of strain (or stress) is applied instead of the constant strain considered by Eshelby in the first order problem. The strain and stress are related outside the heterogeneity by:

$$
\sigma_{i j k}^{\infty}=C_{i j p q}^{0} \varepsilon_{p q k}^{\infty}
$$

At infinity, the equilibrium for the applied remote stress is:

$$
\sigma_{i j j}^{\infty}=C_{i j p q}^{0} \varepsilon_{p q j}^{\infty}=0
$$


$\varepsilon_{i j k}^{\infty}$ and $\sigma_{i j k}^{\infty}$ being symmetric with respect to indices $i$ and $j$ and accounting for the equilibrium condition (4), we deduce that they both depend on 15 independent coefficients.

The strain disturbance due to the application of $\varepsilon_{i j k}^{\infty}$ at infinity can be obtained by considering the following appropriate inclusion problem: an ellipsoidal domain, defined by (1), is subjected to a prescribed eigenstrain of the form $\varepsilon_{i j}^{*}(x)=e_{i j k} x_{k}$ (with $e_{i j k}=e_{j i k}$ ). As shown in [19], [13] and also in [1] for the more general case of an anisotropic matrix, the solution is defined by a linear strain within the inclusion:

$$
\varepsilon_{i j}(x)=S_{i j k p q r} e_{p q r} x_{k}
$$

where $S_{i j k p q r}$ are the components of a sixth-order Eshelby tensor (to make reference to the common fourth order Eshelby tensor when dealing with constant eigenstrain within the inclusion domain). The expression of the stress components (still within the inclusion) are:

$$
\sigma_{i j}(x)=C_{i j m n}^{0}\left(S_{m n k p q r} e_{p q r}-e_{m n k}\right) x_{k}
$$

The components of the sixth order Eshelby tensor are:

$$
\begin{aligned}
8 \pi\left(1-\nu_{0}\right) S_{i j k p q r}= & \left\{\delta_{i j} \delta_{k r} \delta_{p q}\left[T_{I P R}+2 \nu_{0} I_{I R}\right]\right. \\
& +2 I_{i j p q} \delta_{k r}\left[T_{I J R}+\left(1-\nu_{0}\right)\left(I_{I R}+I_{J R}\right)\right] \\
& +2 I_{p q k r} \delta_{i j} T_{I K R}+2 I_{i j k r} \delta_{p q}\left[T_{I J P}+2 \nu_{0} I_{I J}\right] \\
& +2\left(I_{i j p r} \delta_{k q}+I_{i j q r} \delta_{k p}\right)\left[T_{P Q R}+\left(1-\nu_{0}\right) I_{K R}\right] \\
& \left.+2\left(I_{i j k p} \delta_{q r}+I_{i j k q} \delta_{p r}\right)\left[T_{K P Q}+\left(1-\nu_{0}\right) I_{K R}\right]\right\} a_{R}^{2}
\end{aligned}
$$

In which some specific notations introduced in [16] hase been also used: repeated lower case indices are summed from 1 to 3; upper case indices take on the same values as the corresponding lower case ones but are not summed. For example, in the monomial $a_{i} a_{i} b_{I}$, the repeated indice is $i$ and the upper case indice takes the same value as $i$; it gives: $a_{i} a_{i} b_{I}=a_{1}^{2} b_{1}+a_{2}^{2} b_{2}+a_{3}^{2} b_{3}$. An other example is $a_{i} b_{I}$, here $i$ is not summed and this monomial are the components of a thirst order tensor whose components are: $a_{1} b_{1}, a_{2} b_{2}, a_{3} b_{3}$. In (7), $I_{i j}$ and $T_{i j k}$ are invariant by any permutation of their indices and they are defined in terms of elliptic integrals:

$$
\begin{aligned}
& I_{i j}=2 \pi a_{1} a_{2} a_{3} \int_{0}^{+\infty} \frac{d s}{\left(a_{i}^{2}+s\right)\left(a_{j}^{2}+s\right) \Delta(s)} \\
& T_{i j k}=2 \pi a_{1} a_{2} a_{3} \int_{0}^{+\infty} \frac{s d s}{\left(a_{i}^{2}+s\right)\left(a_{j}^{2}+s\right)\left(a_{k}^{2}+s\right) \Delta(s)}
\end{aligned}
$$


with: $\Delta(s)=\left(a_{1}^{2}+s\right)^{1 / 2}\left(a_{2}^{2}+s\right)^{1 / 2}\left(a_{3}^{2}+s\right)^{1 / 2}$. There are explicit expressions for these integrals when at least two semi-axes are equal (that corresponding to an oblate or a prolate spheroidal inclusion). These expressions are provided in appendix.

Let us now come back to the inhomogeneity problem. Using the equivalent Eshelby inclusion method, the eigenstrain $\varepsilon_{i j}^{*}(x)$ is chosen as:

$$
\varepsilon_{i j}^{*}(x)=Y_{i j p q} \varepsilon_{p q}(x)
$$

where $Y_{i j p q}$ is defined by:

$$
Y_{i j p q}=\alpha \delta_{i j} \delta_{p q} / 3+\beta\left(I_{i j p q}-\delta_{i j} \delta_{p q} / 3\right)
$$

with:

$$
\alpha=1-\frac{k}{k_{0}}, \quad \beta=1-\frac{\mu}{\mu_{0}}
$$

where $k=\lambda+2 \mu / 3$ and $k_{0}=\lambda_{0}+2 \mu_{0} / 3$ are the compressibility moduli of the inhomogeneity and of the infinite medium. The strain field in the ellipsoidal inhomogeneity is also linear according to the vector position and can then be written as $\varepsilon_{i j}(x)=a_{i j k} x_{k}$ where $a_{i j k}$ are the components of a constant thirdorder tensor $\boldsymbol{a}$ which possesses the symmetry with respect to its two first indices. The strain field, solution of the inhomogeneity problem, is the sum of: (i) the prescribed remote strain field $\varepsilon_{i j}(x)=\varepsilon_{i j k}^{\infty} x_{k}$, (ii), the disturbed strain field due to the applied eigenstrain $\varepsilon_{i j}^{*}(x)=e_{i j k} x_{k}$ where $e_{i j k}$ is related to $a_{i j k}$ by $e_{i j k}=Y_{i j p q} a_{p q k}$. It follows that $\boldsymbol{a}$ is solution of the linear system:

$$
\left[I_{i j k p q r}-S_{i j k m n r} Y_{m n p q}\right] a_{p q r}=\varepsilon_{i j k}^{\infty}
$$

in which $I_{i j k p q r}$ are the components of the sixth-order identity tensor given by:

$$
I_{i j k p q r}=\frac{1}{2}\left(\delta_{i p} \delta_{j q}+\delta_{i q} \delta_{j p}\right) \delta_{k r}
$$

The resolution of equation (12) involves the inversion of a sixth order tensor, that constitutes the main difficulty when dealing with arbitrary ellipsoidal inhomogeneities. This equation can be also written by using the applied gradient of stress $\boldsymbol{\sigma}^{\infty}$ as second member in the linear system:

$$
C_{i j u v}^{0}\left[I_{u v k p q r}-S_{u v k m n r} Y_{m n p q}\right] a_{p q r}=\sigma_{i j k}^{\infty}
$$


and can be recast into the following tensorial form:

$$
\mathbb{A} \odot_{3} \boldsymbol{a}=\boldsymbol{\sigma}^{\infty}
$$

where $\mathbb{A}$ is the sixth order tensor whose components are:

$$
A_{i j k p q r}=C_{i j u v}^{0}\left[I_{u v k p q r}-S_{u v k m n r} Y_{m n p q}\right]
$$

and $\odot_{3}$ denotes the inner product on three indices defined by $\left(\mathbb{A} \odot_{3} \boldsymbol{a}\right)_{i j k}=$ $A_{i j k p q r} a_{p q r}$. Tensor $\mathbb{A}$ can be written with the following equivalent form:

$$
\mathbb{A}=\mathbb{C}^{0} \odot_{3}\left(\mathbb{I}-\mathbb{S} \odot_{3} \mathbb{Y}\right)
$$

in which $\mathbb{I}$ is the sixth order identity tensor and $\mathbb{S}$ is the sixth order Eshelby tensor whose components are given by (13) and (7) respectively. In (17), $\mathbb{C}^{0}$ and $\mathbb{Y}$ are also two sixth order tensors; their components are $C_{i j k p q r}^{0}=C_{i j p q}^{0} \delta_{k r}$ and $Y_{i j k p q r}=Y_{i j p q} \delta_{k r}$ in which $Y_{i j p q}$ are given by (10). Obviously, $C_{i j p q}^{0}$ and $Y_{i j p q}$ are associated with fourth order tensors; however the introduction of sixth order tensors is more suitable since the computation of the components of $\mathbb{A}$ can be made in term of those of $\mathbb{C}^{0}, \mathbb{I}, \mathbb{S}$ and $\mathbb{Y}$ by using an irreducible representation with a canonical basis (this is detailed in the next section). Since any third order tensor with previously defined symmetries depends on 18 independent coefficients, the direct inversion of tensor $\mathbb{A}$ involves one of a matrix of dimensions $18 \times 18$. Since the problem has symmetries, it is possible to represent sixth order tensors by using an irreducible basis, which reduces significantly the size of the system.

When dealing with the case of voids, particular attention must be paid since tensor $\mathbb{A}$ is singular and the linear system (15) has no more a unique solution for $\boldsymbol{a}$. This has been already pointed out by [6] (see also [16,18,22]). Indeed, in the case of a cavity we put $k=\mu=0$ in (10), it follows that $\mathbb{Y}=\mathbb{I}$ and tensor $\mathbb{A}$ becomes:

$$
\mathbb{A}=\mathbb{C}^{0} \odot_{3}(\mathbb{I}-\mathbb{S})
$$

Let $\mathcal{A}(\boldsymbol{a})$ being the linear application defined by $\mathcal{A}(\boldsymbol{a}): \boldsymbol{a} \rightarrow \mathbb{A} \odot_{3} \boldsymbol{a}$, the kernel of the linear application $\mathcal{A}(\boldsymbol{a})$ is the space of dimension 3 :

$$
\operatorname{Ker}(\mathcal{A})=\left\{\boldsymbol{a} \mid a_{i j k}=\theta_{i} \eta_{j k}+\theta_{j} \eta_{i k}\right\}
$$


where $\theta_{i}$ are the components of an arbitrary vector and $\eta_{i j}$ is related to the ellipsoid shape by (2). The kernel of $\mathcal{A}$ is associated with some impotent eigenstrains [6] which do not produce a stress field around the inhomogeneity. In the case of voids, the strain $\varepsilon_{i j}(x)=a_{i j k} x_{k}$ is obtained by making a continuation by continuity of the displacement field within the cavity; however, this continuation is not unique, since there exist some displacement fields which produce constant gradients of strain within the void and which are null on its boundary. These displacements read:

$$
u_{i}(x)=\theta_{i}\left(\eta_{p q} x_{p} x_{q}-1\right)
$$

where the term $\eta_{p q} x_{p} x_{q}$ takes the value 1 for a point located on the boundary of the ellipsoidal void. The displacement (20) produces the strain $\varepsilon_{i j}(x)=$ $\theta_{i} \eta_{j k} x_{k}+\theta_{j} \eta_{i k} x_{k}$ and then the constant gradient of strain $\theta_{i} \eta_{j k}+\theta_{j} \eta_{i k}$.

On another hand, by examining the equilibrium condition for the stress field solution of the inclusion problem, that corresponding to $j=k$ in relation (6), we observe that:

$$
A_{i j j p q r}=0
$$

It follows that the image of the application $\mathcal{A}$ is defined by :

$$
\operatorname{Im}(\mathcal{A}(\boldsymbol{a}))=\left\{\boldsymbol{b} \mid b_{i j j}=0\right\}
$$

and is of dimension 15 . We conclude that in the case of voids, the linear system degenerates into a problem of dimension 15.

\section{Case of spheroidal inhomogeneities: decomposition on a canoni- cal basis for transversely isotropic sixth order tensor}

The case of spheroidal inhomogeneities corresponds to $a_{1}=a_{2}$. In this case, the sixth order Eshelby tensor, and then also tensor $\mathbb{A}$, is invariant by any rotation around the axis $O x_{3}$ and by the reflection related to the plane $O x_{1} x_{2}$. This corresponds to the transversely isotropic symmetry, for which decomposition of a sixth order tensor on a canonical basis is possible [12].

Any transversely isotropic sixth order $\mathbb{A}$ can be decomposed as follows:

$$
\mathbb{A}=\sum_{n=1}^{n=31} a_{n} \mathbb{T}_{n}
$$


where the $a_{n}$ are the components of the tensor $\mathbb{A}$ in the basis $\left(\mathbb{T}_{1}, \ldots, \mathbb{T}_{31}\right)$. The definitions for tensors $\mathbb{T}_{n}$ can be found in [12]. The set of tensors $\left(\mathbb{T}_{1}, \ldots, \mathbb{T}_{31}\right)$ is constituted of 5 groups which are independent for the composition $\odot_{3}$. These groups are $\left\{\mathbb{T}_{1}\right\},\left\{\mathbb{T}_{2}\right\},\left\{\mathbb{T}_{3}, \ldots, \mathbb{T}_{6}\right\},\left\{\mathbb{T}_{7}, \ldots, \mathbb{T}_{15}\right\},\left\{\mathbb{T}_{16}, \ldots, \mathbb{T}_{31}\right\}$, and define subspaces whose dimensions are respectively: 1, 1, 4, 9, 25. It follows that an alternative representation for $\mathbb{A}$ is:

$$
\mathbb{A}=\left\{a_{1}, a_{2}, A_{1}, A_{2}, A_{3}\right\}
$$

in which $A_{1}, A_{2}$ et $A_{3}$ are matrices defined by :

$$
A_{1}=\left[\begin{array}{ll}
a_{3} & a_{4} \\
a_{5} & a_{6}
\end{array}\right], A_{2}=\left[\begin{array}{lll}
a_{7} & a_{8} & a_{9} \\
a_{10} & a_{11} & a_{12} \\
a_{13} & a_{14} & a_{15}
\end{array}\right], A_{3}=\left[\begin{array}{llll}
a_{16} & a_{17} & a_{18} & a_{19} \\
a_{20} & a_{21} & a_{22} & a_{23} \\
a_{24} & a_{25} & a_{26} & a_{27} \\
a_{28} & a_{29} & a_{30} & a_{31}
\end{array}\right]
$$

and is called compact representation of $\mathbb{A}$ since this form is irreducible for such a symmetry.

These notations are very useful for performing the usual tensorial operations. For instance the product between two sixth order tensors $\mathbb{A}$ and $\mathbb{B}$ are obtained by making the product of the elements of their respective compact representation:

$$
\mathbb{A} \odot_{3} \mathbb{B}=\left\{a_{1} b_{1}, a_{2} b_{2}, A_{1} B_{1}, A_{2} B_{2}, A_{3} B_{3}\right\}
$$

and the inverse of $\mathbb{A}$ is:

$$
\mathbb{A}^{-1}=\left\{\frac{1}{a_{1}}, \frac{1}{a_{2}}, A_{1}^{-1}, A_{2}^{-1}, A_{3}^{-1}\right\}
$$

with the following inversibility condition $a_{1} a_{2} \operatorname{det}\left(A_{1}\right) \operatorname{det}\left(A_{2}\right) \operatorname{det}\left(A_{3}\right) \neq 0$. Coming back now to the inhomogeneity problem, the inversion of $\mathbb{A}$ is effected with relation (27), where the expressions of the elements of its compact representation are obtained by the sum and product of tensors $\mathbb{I}, \mathbb{S}$ and $\mathbb{Y}$. The elements of the compact representation of tensor $\mathbb{A}$ given by (17), are:

$$
\begin{aligned}
& a_{1}=2 \mu_{0}\left(1-\beta s_{1}\right), \quad a_{2}=2 \mu_{0}\left(1-\beta s_{2}\right), \quad A_{1}=C_{1}^{0}\left(I_{2}-S_{2} Y_{2}\right), \\
& A_{2}=C_{2}^{0}\left(I_{3}-S_{3} Y_{3}\right), \quad A_{3}=C_{3}^{0}\left(I_{4}-S_{3} Y_{3}\right)
\end{aligned}
$$

in which $I_{2}, I_{3}$ and $I_{4}$ are the identity matrices of dimension 2, 3 and 4 respectively. Matrices $S_{2}, S_{3}, S_{4}$ and $Y_{2}, Y_{3}, Y_{4}$ and also $C_{1}^{0}, C_{2}^{0}, C_{3}^{0}$ can be found in 
appendix.

The relationship $\boldsymbol{\sigma}^{\infty}=\mathbb{A} \odot_{3} \boldsymbol{a}$ can be put into the form:

$$
\begin{gathered}
\boldsymbol{\sigma}_{\mathrm{I}}^{\infty}=a_{1} \boldsymbol{a}_{\mathrm{I}}, \quad \sigma_{\mathrm{II}}^{\infty}=a_{2} a_{\mathrm{II}}, \quad\left[\begin{array}{l}
\boldsymbol{\sigma}_{\mathrm{II}}^{\infty} \\
\boldsymbol{\sigma}_{\mathrm{IV}}^{\infty}
\end{array}\right]=A_{1}\left[\begin{array}{l}
\boldsymbol{a}_{\mathrm{III}} \\
\boldsymbol{a}_{\mathrm{IV}}
\end{array}\right], \\
{\left[\begin{array}{l}
\sigma_{\mathrm{V}}^{\infty} \\
\sigma_{\mathrm{VI}}^{\infty} \\
\sigma_{\mathrm{VII}}^{\infty}
\end{array}\right]=A_{2}\left[\begin{array}{l}
a_{\mathrm{V}} \\
a_{\mathrm{VI}} \\
a_{\mathrm{VII}}
\end{array}\right],\left[\begin{array}{l}
\boldsymbol{\sigma}_{\mathrm{VIII}}^{\infty} \\
\boldsymbol{\sigma}_{\mathrm{IX}}^{\infty} \\
\boldsymbol{\sigma}_{\mathrm{X}}^{\infty} \\
\boldsymbol{\sigma}_{\mathrm{XI}}^{\infty}
\end{array}\right]=A_{3}\left[\begin{array}{l}
\boldsymbol{a}_{\mathrm{VIII}} \\
\boldsymbol{a}_{\mathrm{IX}} \\
\boldsymbol{a}_{\mathrm{XI}}
\end{array}\right]}
\end{gathered}
$$

where the following notations have been used:

$$
\begin{aligned}
& \boldsymbol{a}_{\mathrm{I}}=\left[\begin{array}{l}
\left(a_{112}+2 a_{121}-a_{222}\right) / 4 \\
\left(a_{221}+2 a_{122}-a_{111}\right) / 4
\end{array}\right], \quad a_{\mathrm{II}}=a_{132}-a_{231}, \\
& \boldsymbol{a}_{\mathrm{III}}=\left[\begin{array}{c}
\left(a_{113}-a_{223}\right) / 2 \\
a_{123}
\end{array}\right], \quad \boldsymbol{a}_{\mathrm{IV}}=\left[\begin{array}{c}
a_{131}-a_{232} \\
a_{132}+a_{231}
\end{array}\right], \\
& a_{\mathrm{V}}=\left(a_{113}+a_{223}\right) / 2, \quad a_{\mathrm{VI}}=a_{131}+a_{232}, \quad a_{\mathrm{VII}}=a_{333}, \\
& \boldsymbol{a}_{\mathrm{VIII}}=\left[\begin{array}{l}
\left(a_{111}-a_{221}\right) / 2+a_{122} \\
\left(a_{222}-a_{112}\right) / 2+a_{121}
\end{array}\right], \quad \boldsymbol{a}_{\mathrm{IX}}=\left[\left(\begin{array}{l}
\left(a_{111}+3 a_{221}\right) / 4-a_{122} / 2 \\
\left.a_{222}+3 a_{112}\right) / 4-a_{121} / 2
\end{array}\right],\right. \\
& \boldsymbol{a}_{\mathrm{X}}=\left[\begin{array}{l}
a_{331} \\
a_{332}
\end{array}\right], \quad \boldsymbol{a}_{\mathrm{XI}}=\left[\begin{array}{l}
a_{133} \\
a_{233}
\end{array}\right]
\end{aligned}
$$

and the same notations are considered for the applied stress gradient $\boldsymbol{\sigma}^{\infty}$. It provides a direct link between the components of $\boldsymbol{\sigma}^{\infty}$ and those of tensor $\boldsymbol{a}$ when they are written in the cartesian frame aligned with the axis of transverse isotropy (axis $O x_{3}$ ).

The computation of the components of the strain for an interior point as a function of the applied remote stress gradient then involves the inversion of two scalars and matrices of dimension 2, 3 and 4 . With this compact representation, the size of the problem is significantly reduced. When the case of voids is considered, the problem can be again reduced since, as mentioned in the previous section, the problem initially of dimension 18 degenerates to a problem of dimension 15.

Let us examine the case of void more precisely. The elements of the compact representation of $\mathbb{A}$ are obtained by putting $\beta=1$ and $Y_{1}, Y_{2}$ and $Y_{3}$ are the identity matrices. This leads to: 


$$
\begin{aligned}
& a_{1}=2 \mu_{0}\left(1-s_{1}\right), \quad a_{2}=2 \mu_{0}\left(1-s_{2}\right), \quad A_{1}=C_{1}^{0}\left(I_{2}-S_{2}\right) \\
& A_{2}=C_{2}^{0}\left(I_{3}-S_{3}\right), \quad A_{3}=C_{3}^{0}\left(I_{4}-S_{3}\right)
\end{aligned}
$$

$a_{1}, a_{2}$ and all the components of $A_{1}, A_{2}, A_{3}$ are given in appendix. The components of matrices $A_{2}$ and $A_{3}$ which are formally given by (25) comply with the following relations:

$$
\begin{aligned}
& \left\{\begin{array} { l } 
{ a _ { 9 } \epsilon ^ { 2 } + a _ { 8 } = 0 } \\
{ a _ { 1 2 } \epsilon ^ { 2 } + a _ { 1 1 } = 0 \text { group (a) } } \\
{ a _ { 1 5 } \epsilon ^ { 2 } + a _ { 1 4 } = 0 }
\end{array} \quad \left\{\begin{array}{l}
a_{10}+a_{13}=0 \\
a_{11}+a_{14}=0 \text { group }(\mathrm{b}) \\
a_{12}+a_{15}=0
\end{array}\right.\right. \\
& \left\{\begin{array} { l } 
{ 2 a _ { 1 6 } \epsilon ^ { 2 } + a _ { 1 9 } = 0 } \\
{ 2 a _ { 2 0 } \epsilon ^ { 2 } + a _ { 2 3 } = 0 \text { group (c) } } \\
{ 2 a _ { 2 4 } \epsilon ^ { 2 } + a _ { 2 7 } = 0 } \\
{ 2 a _ { 2 4 } \epsilon ^ { 2 } + a _ { 3 1 } = 0 }
\end{array} \quad \left\{\begin{array}{l}
3 a_{16}+2 a_{20}+2 a_{24}=0 \\
3 a_{17}+2 a_{21}+2 a_{25}=0 \text { group (d) } \\
3 a_{18}+2 a_{22}+2 a_{26}=0 \\
3 a_{19}+2 a_{23}+2 a_{27}=0
\end{array}\right.\right.
\end{aligned}
$$

in which we have introduced the aspect ratio:

$$
\epsilon=\frac{a}{b}
$$

The group of equations (a) and (c) shows that the solution for $\boldsymbol{a}$ is not unique, because the second and the third columns of matrix $A_{2}$ are proportional, as are the first and last columns of $A_{3}$.

In fact, this set of equations contains some impotent strains which read for the spheroidal inhomogeneity:

$$
\begin{aligned}
& \boldsymbol{a}_{\mathrm{I}}=0, \quad a_{\mathrm{II}}=0, \quad \boldsymbol{a}_{\mathrm{III}}=0, \quad \boldsymbol{a}_{\mathrm{IV}}=0, \quad a_{\mathrm{V}}=0, \quad a_{\mathrm{VI}}=\frac{2}{b^{2}} \theta_{3} \\
& a_{\mathrm{VII}}=\frac{2}{a^{2}} \theta_{3}, \quad \boldsymbol{a}_{\mathrm{VIII}}=\frac{2}{b^{2}}\left[\begin{array}{c}
\theta_{1} \\
\theta_{2}
\end{array}\right], \quad \boldsymbol{a}_{\mathrm{IX}}=0, \quad \boldsymbol{a}_{\mathrm{X}}=0, \quad \boldsymbol{a}_{\mathrm{XI}}=\frac{1}{a^{2}}\left[\begin{array}{c}
\theta_{1} \\
\theta_{2}
\end{array}\right]
\end{aligned}
$$

From another point of view, the group of equations (b) and (d) is the detailed expression of the equilibrium condition (21).

By eliminating the equilibrium and the impotent components of $\boldsymbol{a}$ we obtain the following reduced linear system for the spheroidal cavities: 


$$
\begin{aligned}
& \boldsymbol{\sigma}_{\mathrm{I}}^{\infty}=a_{1} \boldsymbol{a}_{\mathrm{I}}, \quad \sigma_{\mathrm{II}}^{\infty}=a_{2} a_{\mathrm{II}}, \quad\left[\begin{array}{l}
\boldsymbol{\sigma}_{\mathrm{III}}^{\infty} \\
\boldsymbol{\sigma}_{\mathrm{IV}}^{\infty}
\end{array}\right]=A_{1}\left[\begin{array}{l}
\boldsymbol{a}_{\mathrm{III}} \\
\boldsymbol{a}_{\mathrm{IV}}
\end{array}\right], \\
& {\left[\begin{array}{l}
\sigma_{\mathrm{V}}^{\infty} \\
\sigma_{\mathrm{VI}}^{\infty}
\end{array}\right]=\bar{A}_{2}\left[\begin{array}{l}
a_{\mathrm{V}} \\
a_{\mathrm{VI}}-\epsilon^{2} a_{\mathrm{VII}}
\end{array}\right], \quad\left[\begin{array}{l}
\boldsymbol{\sigma}_{\mathrm{VIII}}^{\infty} \\
\boldsymbol{\sigma}_{\mathrm{IX}}^{\infty} \\
\boldsymbol{\sigma}_{\mathrm{X}}^{\infty}
\end{array}\right]=\bar{A}_{3}\left[\begin{array}{l}
\boldsymbol{a}_{\mathrm{VIII}}-\epsilon^{2} a_{\mathrm{XI}} \\
\boldsymbol{a}_{\mathrm{IX}} \\
\boldsymbol{a}_{\mathrm{X}}
\end{array}\right]}
\end{aligned}
$$

which corresponds to 15 scalar coefficients.

In addition, the reduced matrices $\bar{A}_{2}$ and $\bar{A}_{3}$ are defined by:

$$
\bar{A}_{2}=\left[\begin{array}{cc}
a_{7} & a_{8} \\
a_{10} & a_{11}
\end{array}\right], \bar{A}_{3}=\left[\begin{array}{lll}
a_{16} & a_{17} & a_{18} \\
a_{20} & a_{21} & a_{22} \\
a_{24} & a_{25} & a_{26}
\end{array}\right]
$$

In the case of void, the resolution of the linear system consists then in the inversion of two scalars $\left(a_{1}\right.$ and $\left.a_{2}\right)$, two matrices of dimension $2\left(A_{1}\right.$ and $\left.\bar{A}_{2}\right)$ and one matrix of dimension $3\left(\bar{A}_{3}\right)$. Explicit solutions are now provided in the case of the penny shaped crack.

\section{Solution for the penny-shaped crack}

The case of a penny-shaped crack is the special case of an oblate spheroidal void when its radius $a$ along the $O x_{3}$ axis vanishes. Taking the limit $\epsilon \rightarrow 0$ in $a_{1}, a_{2}$ and in matrices $A_{1}, \bar{A}_{2}, \bar{A}_{3}$, we obtain the following expressions at the first order:

$$
\begin{aligned}
& a_{1}=2 \mu_{0}-\frac{3 \pi}{16} \frac{7-8 \nu_{0}}{1-\nu_{0}} \epsilon+o(\epsilon), \\
& a_{2}=\frac{3 \mu_{0} \pi}{2} \epsilon+o(\epsilon), \\
& A_{1}=A_{1}^{(0)}+\epsilon A_{1}^{(1)}+o(\epsilon), \\
& \bar{A}_{2}=\bar{A}_{2}^{(0)}+\epsilon \bar{A}_{2}^{(1)}+o(\epsilon), \\
& \bar{A}_{3}=\bar{A}_{3}^{(0)}+\epsilon \bar{A}_{3}^{(1)}+o(\epsilon)
\end{aligned}
$$

with: 


$$
\begin{aligned}
& A_{1}^{(0)}=2 \mu_{0}\left[\begin{array}{cc}
1 & -1 \\
0 & 0
\end{array}\right], A_{1}^{(1)}=\frac{3 \mu_{0} \pi}{8\left(1-\nu_{0}\right)}\left[\begin{array}{cc}
0 & 5-4 \nu_{0} \\
0 & 2\left(2-\nu_{0}\right)
\end{array}\right], \\
& \bar{A}_{2}^{(0)}=\frac{2 \mu_{0}\left(1+\nu_{0}\right)}{1-\nu_{0}}\left[\begin{array}{cc}
1 & -1 \\
0 & 0
\end{array}\right], \bar{A}_{2}^{(1)}=\frac{3 \mu_{0} \pi}{4\left(1-\nu_{0}\right)}\left[\begin{array}{cc}
0 & 3+2 \nu_{0} \\
0 & 2
\end{array}\right] \\
& \bar{A}_{3}^{(0)}=\frac{2 \mu_{0}}{1-\nu_{0}}\left[\begin{array}{ccc}
1-\nu_{0} & 0 & 0 \\
\nu_{0} & 1+\nu_{0} & 0 \\
0 & 0 & 0
\end{array}\right], \\
& \bar{A}_{3}^{(1)}=\frac{3 \mu_{0} \pi}{16\left(1-\nu_{0}\right)}\left[\begin{array}{ccc}
-2\left(5-4 \nu_{0}\right) & -6 & 2\left(1-2 \nu_{0}\right) \\
-\left(1+\nu_{0}\right) & -\left(3+8 \nu_{0}\right) & \left(1+6 \nu_{0}\right) \\
4 & 4\left(1+2 \nu_{0}\right) & 4
\end{array}\right]
\end{aligned}
$$

The limit $\epsilon \rightarrow 0$ corresponds to two very close circular surface across which a jump of the displacement field is generated. This jump can be deduced from the interior point solution for the strain. Indeed, when the limit $\epsilon \rightarrow 0$ is taken, the strain can be expanded as a power series in $\epsilon$ :

$$
\boldsymbol{a}=\frac{1}{\epsilon} \boldsymbol{a}^{(-1)}+\boldsymbol{a}^{(0)}+\epsilon \boldsymbol{a}^{(1)}+\ldots
$$

where the dominent term $\boldsymbol{a}^{(-1)}$ is associated with the singularity. Accounting for the decomposition (24) and collecting all the terms with the same power in $\epsilon$ in the linear system (35), we obtain a hierarchy of problems for all the components of $\boldsymbol{a}$ at every order. The resolution of each linear system leads to the following expressions for the dominant terms:

$$
\begin{aligned}
& \boldsymbol{a}_{\mathrm{I}}^{(-1)}=0, \quad a_{\mathrm{II}}^{(-1)}=\frac{2}{3 \mu_{0} \pi} \sigma_{\mathrm{II}}^{\infty}, \quad \boldsymbol{a}_{\mathrm{III}}^{(-1)}=\boldsymbol{a}_{\mathrm{IV}}^{(-1)}=\frac{4\left(1-\nu_{0}\right)}{3 \mu_{0} \pi\left(2-\nu_{0}\right)} \boldsymbol{\sigma}_{\mathrm{IV}}^{\infty}, \\
& a_{\mathrm{V}}^{(-1)}=a_{\mathrm{VI}}^{(-1)}=\frac{2\left(1-\nu_{0}\right)}{3 \mu_{0} \pi} \sigma_{\mathrm{VI}}^{\infty}, \quad \boldsymbol{a}_{\mathrm{VIII}}^{(-1)}=\boldsymbol{a}_{\mathrm{IX}}^{(-1)}=0, \quad \boldsymbol{a}_{\mathrm{X}}^{(-1)}=\frac{4\left(1-\nu_{0}\right)}{3 \mu_{0} \pi} \boldsymbol{\sigma}_{\mathrm{X}}^{\infty}
\end{aligned}
$$

The terms $\boldsymbol{a}_{\mathrm{VII}}^{(-1)}$ and $\boldsymbol{a}_{\mathrm{XI}}^{(-1)}$, which are associated to impotent strains, remain undetermined. From (40) with definitions (30), we obtain the following expressions for the components of $\boldsymbol{a}$ in the cartesian frame: 


$$
\begin{aligned}
& a_{111}^{(-1)}=a_{112}^{(-1)}=a_{121}^{(-1)}=a_{122}^{(-1)}=a_{221}^{(-1)}=a_{222}^{(-1)}=0 \\
& a_{131}^{(-1)}=\frac{1-\nu_{0}}{3 \pi \mu_{0}\left(2-\nu_{0}\right)}\left[\left(4-\nu_{0}\right) \sigma_{131}^{\infty}-\nu_{0} \sigma_{232}^{\infty}\right] \\
& a_{132}^{(-1)}=\frac{1}{3 \pi \mu_{0}\left(2-\nu_{0}\right)}\left[\left(4-3 \nu_{0}\right) \sigma_{132}^{\infty}-\nu_{0} \sigma_{231}^{\infty}\right] \\
& a_{231}^{(-1)}=\frac{1}{3 \pi \mu_{0}\left(2-\nu_{0}\right)}\left[\left(4-3 \nu_{0}\right) \sigma_{231}^{\infty}-\nu_{0} \sigma_{132}^{\infty}\right] \\
& a_{232}^{(-1)}=\frac{1-\nu_{0}}{3 \pi \mu_{0}\left(2-\nu_{0}\right)}\left[\left(4-\nu_{0}\right) \sigma_{232}^{\infty}-\nu_{0} \sigma_{131}^{\infty}\right] \\
& a_{331}^{(-1)}=\frac{4\left(1-\nu_{0}\right)}{3 \mu_{0} \pi} \sigma_{331}^{\infty}, \quad a_{332}^{(-1)}=\frac{4\left(1-\nu_{0}\right)}{3 \mu_{0} \pi} \sigma_{332}^{\infty} \\
& a_{113}^{(-1)}=2 a_{131}^{(-1)}, \quad a_{223}^{(-1)}=2 a_{232}^{(-1)}, \quad a_{123}^{(-1)}=a_{132}^{(-1)}+a_{231}^{(-1)}
\end{aligned}
$$

The strain field being determined for the interior points, one can deduce the solution for the displacement at the surface of the crack. The strain field being linear with respect to the coordinates, the components of the displacement field for the interior points are:

$$
u_{i}=U_{i}+\frac{1}{2} K_{i j k} x_{j} x_{k}
$$

The components $K_{i j k}$ can be easily computed from:

$$
a_{i j k}=\frac{1}{2}\left(u_{i, j k}+u_{j, i k}\right)=\frac{1}{2}\left(K_{i j k}+K_{j i k}\right)
$$

which, by inversion, leads to:

$$
K_{i j k}=a_{i j k}+a_{i k j}-a_{j k i}
$$

It is not necessary to compute the constants $U_{i}$ for $i=1 . .3$ since they do not enter in the expression of the displacement jump vector. Let us now introduce the cylindrical coordinates $(r, \theta, z)$ centered at the origin and such that $z \equiv$ $x_{3}$. The radial coordinate $r$ denotes the distance between the crack center and a current position. For a given value of $r$ and $\theta$ we can define the two opposite positions $z_{+}$and $z_{-}$on the surface of the spheroid (see figure 1). Those positions are:

$$
z_{+}=\epsilon \sqrt{b^{2}-r^{2}}, \quad z_{-}=-\epsilon \sqrt{b^{2}-r^{2}}
$$


The jump of displacement across the crack can be obtained by comparing the value of the displacements for two opposite points taken on the surface of the spheroid, then by taking the limit $\epsilon \rightarrow 0$ :

$$
[\underline{u}]=\lim _{\epsilon \rightarrow 0}\left[\underline{u}\left(r, \theta, \epsilon \sqrt{b^{2}-r^{2}}\right)-\underline{u}\left(r, \theta,-\epsilon \sqrt{b^{2}-r^{2}}\right)\right]
$$

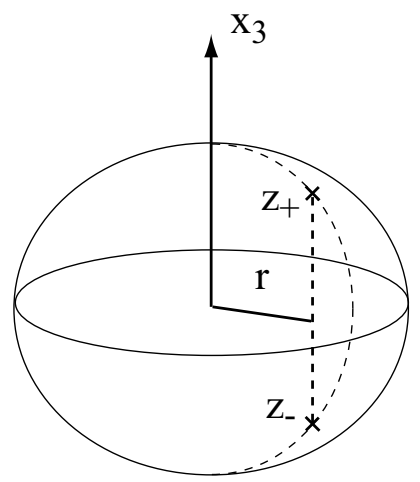

Fig. 1. Radial coordinate and heights $z_{+}$and $z_{-}$for two opposite points taken on the surface of a spheroid

Denoting the displacement jump by:

$$
\Delta \underline{u}=\underline{u}\left(r, \theta, \epsilon \sqrt{b^{2}-r^{2}}\right)-\underline{u}\left(r, \theta,-\epsilon \sqrt{b^{2}-r^{2}}\right)
$$

It can be found, from (42) together with (44), that:

$$
\begin{aligned}
& \Delta u_{1}=2 r \epsilon \sqrt{b^{2}-r^{2}}\left[a_{113} \cos (\theta)+\left(a_{132}+a_{123}-a_{231}\right) \sin (\theta)\right] \\
& \Delta u_{2}=2 r \epsilon \sqrt{b^{2}-r^{2}}\left[\left(a_{123}+a_{231}-a_{132}\right) \cos (\theta)+a_{223} \sin (\theta)\right] \\
& \Delta u_{3}=2 r \epsilon \sqrt{b^{2}-r^{2}}\left[\left(a_{331} \cos (\theta)+a_{332} \sin (\theta)\right]\right.
\end{aligned}
$$

Finally, replacing the components of $a_{i j k}$ by (39) together with (41) and taking the limit $\epsilon \rightarrow 0$, we obtain: 


$$
\begin{gathered}
{\left[u_{1}\right]=\frac{4}{3 \pi \mu_{0}\left(2-\nu_{0}\right)} r \sqrt{b^{2}-r^{2}}\left[\left(1-\nu_{0}\right)\left(\left(4-\nu_{0}\right) \sigma_{131}^{\infty}-\nu_{0} \sigma_{232}^{\infty}\right) \cos (\theta)\right.} \\
\left.+\left(\left(4-3 \nu_{0}\right) \sigma_{132}^{\infty}-\nu_{0} \sigma_{231}^{\infty}\right) \sin (\theta)\right] \\
{\left[u_{2}\right]=\frac{4}{3 \pi \mu_{0}\left(2-\nu_{0}\right)} r \sqrt{b^{2}-r^{2}}\left[\left(4-3 \nu_{0}\right) \sigma_{231}^{\infty}-\nu_{0} \sigma_{132}^{\infty}\right) \cos (\theta)} \\
\left.+\left(1-\nu_{0}\right)\left(\left(4-\nu_{0}\right) \sigma_{232}^{\infty}-\nu_{0} \sigma_{131}^{\infty}\right) \sin (\theta)\right] \\
{\left[u_{3}\right]=\frac{8\left(1-\nu_{0}\right)}{3 \pi \mu_{0}} r \sqrt{b^{2}-r^{2}}\left[\sigma_{331}^{\infty} \cos (\theta)+\sigma_{332}^{\infty} \sin (\theta)\right]}
\end{gathered}
$$

Note that the solution at the first order shows that the displacement jump vector depends only on the stress components acting on the plane of the crack i.e. $\sigma_{13}^{\infty}, \sigma_{23}^{\infty}$ and $\sigma_{33}^{\infty}$. When a stress gradient is applied at infinity, the solution for the sliding mode gives a dependence of $\left[u_{1}\right]$ and $\left[u_{2}\right]$ with the derivatives of the shear stress components along the $x_{1}$ and $x_{2}$ directions. Considering the opening mode, $\left[u_{3}\right]$, it depends on the derivatives of the normal stress along the $x_{1}$ and $x_{2}$ directions.

\section{Conclusion}

In this paper we have derived the displacement jump vector occurring across a penny-shaped crack embedded in an infinite elastic matrix and subjected to a remote gradient of strain, extending older results corresponding to displacement jumps induced by a constant strain at infinity. The method takes advantage of the problem of the inclusion subjected to a polarization that is linear with the coordinates. The problem is thus reduced to the inversion of a sixth order tensor. This inversion is formally equivalent to the resolution of a system of dimension 18. For the case of a spheroidal inhomogeneity, the problem has been proved to be greatly reduced by using a canonical basis for transversely isotropic sixth order tensors. More particularly, attention has been focused on cavities for which the problem becomes singular which is attributed to the presence of impotent strains which remain undetermined. Eliminating the impotent strains leads to a reduced linear system which has been also formulated for the case of cavities.

Finally, the particular case of penny-shaped cracks is studied and the closed form solution for the crack opening displacement has been provided. This extends the classical solution for the jump induced across a penny-shaped crack when a constant strain field is applied at infinity. 


\section{References}

[1] Asaro, R. J. \& Barnett, D. M. (1975) The non-uniform transformation strain problem for an anisotropic ellipsoidal inclusion. J. Mech. Phys. Solids, 1, 77-83.

[2] Benedikt, B., Lewis \& M. , Rangaswamy P. (2006) On elastic interactions between spherical inclusions by the equivalent inclusion method. Comput. Mater. Science, 37, 380-392.

[3] Eshelby, J.D. (1957) The determination of the elastic field of an ellipsoidal inclusion and related problem. Proc. R. Soc. London A, 241, 376-396.

[4] Eshelby, J.D. (1959) The elastic field outside an ellipsoidal inclusion. Proc. Roy. Soc. London A. 252, 561-569.

[5] Eshelby, J.D. (1960) Progress in solid mechanics: Vol. 1. Edited by I. N. Sneddon and R. Hill, North-Holland Publishing Company, Amsterdam.

[6] Furuhashi, R. \& Mura, T. (1979) On the equivalent inclusion method and impotent eigenstrains. J. Elasticity, 9, 263-270.

[7] Gourgiotis P.A. \& Georgiadis, H.G. (2009) Plane-strain crack problems in microstructured solids governed by dipolar gradient elasticity. J. Mech. Phys. Solids 57(11), 1898-1920.

[8] Kachanov, M., Shafiro, B. \& Tsukrov, I. (2003) Handbook of elasticity solutions. KLuwer Academic Publishers.

[9] Mihai, I.C. \& Jefferson, A.D. (2011) A material model for cementitious composite materials with an exterior point Eshelby microcracked initiation criterion. Int. J. Solids Struct. 24(1), 3312-3325.

[10] Monchiet, V. \& Bonnet, G. (2011) Inversion of higher order isotropic tensors with minor symmetries and solution of higher order heterogeneity problems. Proc. Roy. Soc. London. 467(2126), 314-332.

[11] Monchiet, V., Gruescu, C., Cazacu, O. \& Kondo, D. (2012) A micromechanical approach of crack-induced damage in orthotropic media: Application to brittle matrix composite Eng. Frac. Mech., 83, 40-53.

[12] Monchiet, V \& Bonnet, G. (2013) Algebra of transversely isotropic sixth order tensors and solutions to higher order homogenization problems. J. Elasticity, 110(2), 159-183.

[13] Moschovidis, Z.A. (1975a) Two ellipsoidal inhomogeneities and related problems treated by the equivalent inclusion method, Ph.D. thesis, Northwestern University, Illinois.

[14] Moschovidis, Z.A. \& Mura T. (1975b) Two-ellipsoidal inhomogeneities by the equivalent inclusion method, ASME J. Appl. Mech., 42, 847-852. 
[15] Mühlich, U., Zybell, L., Hütter, G. \& Kuna, M.A. (2013) first-order strain gradient damage model for simulating quasi-brittle failure in porous elastic solids. Arch. Appl. Mech., 83(6), 955-967.

[16] Mura, T. (1987) Micromechanics of Defects in Solids. Dordrecht: Martinus Nijhoff.

[17] Nemat-Nasser, S. \& Hori, M. (1999) Micromechanics: Overall Properties of Heterogeneous Materials. North- Holland, Amsterdam.

[18] Nyashin, Y., Lokhov, V. \& Ziegler, F. (2005) Decomposition method in linear elastic problems with eigenstrain. Z. Angew. Math. Mech., 85(8), 557-570.

[19] Sendeckyj, G.P. (1967) Ellipsoidal Inhomogeneity Problem. Ph.D. dissertation, Northwestern University, Evanston, IL.

[20] Shodja, H.M. \& Sarvestani, A.S. (2001) Elastic fields in double inhomogeneity by the equivalent inclusion method. J. Appl. Mech. 68(3), 3-10.

[21] Shodja, H.M., Rad, I.Z. \& Soheilifard, R. (2003) Interacting cracks and ellipsoidal inhomogeneities by the equivalent inclusion method. J. Mech. Phys. Solids. 51, 945-960.

[22] Shodja, H.M. \& Shokrolahi-Zadeh, B. (2007) Ellipsoidal domains: piecewise non uniform and impotent eigenstrain fields. J. Elasticity, 86, 1-18.

[23] Walpole, L.J. (1981) Elastic behavior of composite materials : theoretical foundations. Advances in App. Mech., 21, 169-243.

[24] Walpole, L.J. (1984) Fourth-rank tensors of the thirthy-two crystal classes: multiplication table. Proc. R. Soc. Lond. A., 391, 149-179.

\section{A Elements of the compact representation of tensors $\mathbb{S}, \mathbb{Y}, \mathbb{C}^{0}$ and $\mathbb{A}$}

The elliptic integrals, given by relation (8), are given by: 


$$
\begin{aligned}
& I_{11}=I_{22}=I_{12}=\frac{\pi\left(3(\eta-1)+2 \epsilon^{2}\right)}{2 b^{2}\left(\epsilon^{2}-1\right)}, \quad I_{13}=I_{23}=\frac{2 \pi(1-3 \eta)}{b^{2}\left(\epsilon^{2}-1\right)} \\
& I_{33}=\frac{4 \pi\left(3 \eta \epsilon^{2}-1\right)}{3 b^{2} \epsilon^{2}\left(\epsilon^{2}-1\right)} \\
& T_{111}=T_{112}=T_{122}=T_{222}=\frac{\pi\left(6 \epsilon^{2}(2-3 \eta)+3(\eta-1)-4 a^{4}\right)}{12 b^{2}\left(\epsilon^{2}-1\right)^{2}} \\
& T_{113}=T_{123}=T_{223}=\frac{\pi\left(3(\eta-1)+12 \eta \epsilon^{2}-2 \epsilon^{2}\right)}{2 b^{2}\left(\epsilon^{2}-b^{2}\right)^{2}} \\
& T_{133}=T_{233}=\frac{2 \pi\left(5-9 \eta-6 \epsilon^{2} \eta\right)}{3\left(\epsilon^{2}-1\right)^{2}} \\
& T_{333}=\frac{4 \pi\left(3 \epsilon^{2}(5 \eta-1)-2\right)}{15 b^{2} \epsilon^{2}\left(\epsilon^{2}-1\right)^{2}}
\end{aligned}
$$

where $\eta$ is defined by:

$$
\eta= \begin{cases}\frac{\epsilon}{\left(\epsilon^{2}-1\right)^{3 / 2}} \operatorname{arctanh}\left\{\frac{\sqrt{\epsilon^{2}-1}}{\epsilon}\right\}-\frac{1}{\epsilon^{2}-1} & \text { (prolate spheroid) } \\ -\frac{\epsilon}{\left(1-\epsilon^{2}\right)^{3 / 2}} \arctan \left\{\frac{\sqrt{1-\epsilon^{2}}}{\epsilon}\right\}+\frac{1}{1-\epsilon^{2}} & \text { (oblate spheroid) }\end{cases}
$$

The introduction of $\eta$ allows to produce various equations which are valid for both the case of an oblate spheroid $(\epsilon \leq 1)$ and a prolate spheroid $(\epsilon \geq 1)$. The components of the sixth order Eshelby tensor, written in the canonical basis, are: 


$$
\begin{aligned}
& s_{1}=\frac{\left(6-8 \nu_{0}\right)(1-p)-q}{8\left(1-\nu_{0}\right)}, \quad s_{2}=p, \\
& s_{3}=\frac{q+8 p\left(1-\nu_{0}\right)}{4\left(1-\nu_{0}\right)} \epsilon^{2}, \quad s_{4}=\frac{q+4 p\left(1-\nu_{0}\right)}{4\left(1-\nu_{0}\right)} \\
& s_{5}=\frac{q+4 p\left(1-\nu_{0}\right)}{2\left(1-\nu_{0}\right)} \epsilon^{2}, \quad s_{6}=\frac{1}{2}+\frac{q+p\left(1-\nu_{0}\right)}{2\left(1-\nu_{0}\right)} \text {, } \\
& s_{7}=\frac{q+4 p}{2\left(1-\nu_{0}\right)} \epsilon^{2}, \quad s_{8}=\frac{q+2 p\left(1-\nu_{0}\right)}{2\left(1-\nu_{0}\right)}, \\
& s_{9}=-\frac{q+2 p\left(1-\nu_{0}\right)}{2\left(1-\nu_{0}\right)} \epsilon^{2}, \quad s_{10}=\frac{q+2 p\left(1+\nu_{0}\right)}{1-\nu_{0}} \epsilon^{2}, \\
& s_{11}=1+\frac{q}{\left(1-\nu_{0}\right)}, \quad s_{12}=-\frac{q}{1-\nu_{0}} \epsilon^{2}, \\
& s_{13}=\frac{2 \nu_{0}-\left(q+2 p+4 p \nu_{0}\right) \epsilon^{2}}{1-\nu_{0}}, \quad s_{14}=-\frac{q+2 p \nu_{0}}{\left(1-\nu_{0}\right)}, \\
& s_{15}=1+\frac{q+2 p \nu_{0}}{1-\nu_{0}} \epsilon^{2}, \quad s_{16}=1-\frac{q+4 p\left(1-\nu_{0}\right)}{4\left(1-\nu_{0}\right)} \text {, } \\
& s_{17}=\frac{-q-2 p+2}{4\left(1-\nu_{0}\right)}, \quad s_{18}=\frac{q-2 \nu_{0}(p-1)}{4\left(1-\nu_{0}\right)} \text {, } \\
& s_{19}=\frac{q+4 p\left(1-\nu_{0}\right)}{2\left(1-\nu_{0}\right)} \epsilon^{2}, \quad s_{20}=-\frac{q}{8\left(1-\nu_{0}\right)}, \\
& s_{21}=-\frac{q+2(p-1)}{8\left(1-\nu_{0}\right)}, \quad s_{22}=\frac{q-2 \nu_{0}(p-1)}{8\left(1-\nu_{0}\right)}, \\
& s_{23}=\frac{q}{1-\nu_{0}} \epsilon^{2}, \quad s_{24}=\frac{q+2 p \nu_{0}}{2\left(1-\nu_{0}\right)} \\
& s_{25}=\frac{q+4 p \nu_{0}}{2\left(1-\nu_{0}\right)}, \quad s_{26}=\frac{-q+2 p\left(1-\nu_{0}\right)}{2\left(1-\nu_{0}\right)}, \\
& s_{27}=-\frac{q+2 p \nu_{0}}{1-\nu_{0}} \epsilon^{2}, \quad s_{28}=-\frac{-q+p\left(\nu_{0}-3\right)}{2\left(1-\nu_{0}\right)} \text {, } \\
& s_{29}=\frac{q+2 p\left(1+\nu_{0}\right)}{2\left(1-\nu_{0}\right)}, \quad s_{30}=-\frac{q}{2\left(1-\nu_{0}\right)} \\
& s_{31}=1+\frac{-q+p\left(\nu_{0}-3\right)}{1-\nu_{0}} \epsilon^{2}
\end{aligned}
$$

in which $p$ and $q$ are two functions of the aspect ratio $\epsilon$ given by:

$$
p=\frac{1-3 \eta}{2\left(\epsilon^{2}-1\right)}, \quad q=\frac{3(\eta-1)+12 \eta \epsilon^{2}-2 \epsilon^{2}}{2\left(\epsilon^{2}-b^{2}\right)^{2}}
$$

In the case of cracks, the expansion series of $p$ and $q$ at $\epsilon=0$ is: 


$$
p=1-\frac{3 \pi}{4} \epsilon+o(\epsilon), \quad q=-\frac{3 \pi}{4} \epsilon+o(\epsilon)
$$

The element of the compact representation of $\mathbb{Y}$ are $\left\{\beta, \beta, Y_{1}, Y_{2}, Y_{3}\right\}$ with:

$$
\begin{aligned}
& Y_{1}=\left[\begin{array}{ll}
\beta & 0 \\
0 & \beta
\end{array}\right], \quad Y_{2}=\frac{1}{3}\left[\begin{array}{ccc}
2 \alpha+\beta & 0 & \alpha-\beta \\
0 & 3 \beta & 0 \\
\alpha-\beta & 0 & 2 \alpha+\beta
\end{array}\right], \\
& Y_{3}=\frac{1}{3}\left[\begin{array}{cccc}
3 \beta & 0 & 0 & 0 \\
\alpha-\beta & (2 \alpha+\beta) & \alpha-\beta & 0 \\
\alpha-\beta & 2(\alpha-\beta) & 2 \alpha+\beta & 0 \\
0 & 0 & 0 & 3 \beta
\end{array}\right]
\end{aligned}
$$

The element of the compact representation of $\mathbb{C}^{0}$ are $\left\{2 \mu_{0}, 2 \mu_{0}, C_{1}^{0}, C_{2}^{0}, C_{3}^{0}\right\}$ with:

$$
\begin{aligned}
& C_{1}^{0}=2 \mu_{0}\left[\begin{array}{ll}
1 & 0 \\
0 & 1
\end{array}\right], C_{2}^{0}=\frac{2 \mu_{0}}{1-2 \nu_{0}}\left[\begin{array}{ccc}
1 & 0 & \nu_{0} \\
0 & 1-2 \nu_{0} & 0 \\
\nu_{0} & 0 & 1
\end{array}\right], \\
& C_{3}^{0}=\frac{2 \mu_{0}}{1-2 \nu_{0}}\left[\begin{array}{cccc}
1-2 \nu_{0} & 0 & 0 & 0 \\
\nu_{0} & 1 & \nu_{0} & 0 \\
\nu_{0} & 2 \nu_{0} & 1 & 0 \\
0 & 0 & 0 & 1-2 \nu_{0}
\end{array}\right]
\end{aligned}
$$

In the case of a cavity, the components of $\mathbb{A}$ are: 


$$
\begin{aligned}
& a_{1}=\mu_{0} \frac{2+q+\left(6-8 \nu_{0}\right) p}{4\left(1-\nu_{0}\right)} \quad a_{2}=2 \mu_{0}(1-p) \\
& a_{3}=2 \mu_{0}-\mu_{0} \frac{q+8 p\left(1-\nu_{0}\right)}{2\left(1-\nu_{0}\right)} \epsilon^{2} \quad a_{4}=-\mu_{0} \frac{q+4 p\left(1-\nu_{0}\right)}{2\left(1-\nu_{0}\right)} \\
& a_{5}=-\mu_{0} \frac{q+4 p\left(1-\nu_{0}\right)}{1-\nu_{0}} \epsilon^{2} \quad a_{6}=\mu_{0}-\mu_{0} \frac{q+p\left(1-\nu_{0}\right)}{1-\nu_{0}} \\
& a_{7}=-\mu_{0} \frac{\left(2\left(1+\nu_{0}\right)\left(2 p \epsilon^{2}-1\right)+q \epsilon^{2}\right)}{1-\nu_{0}} \quad a_{8}=-\mu_{0} \frac{q+2 p\left(1+\nu_{0}\right)}{1-\nu_{0}} \\
& a_{9}=\mu_{0} \frac{q+2 p\left(1+\nu_{0}\right)}{1-\nu_{0}} \epsilon^{2} \quad a_{10}=-2 \mu_{0} \frac{q+2 p\left(1+\nu_{0}\right)}{1-\nu_{0}} \epsilon^{2} \\
& a_{11}=-2 \mu_{0} \frac{q}{1-\nu_{0}} \quad a_{12}=2 \mu_{0} \frac{q}{1-\nu_{0}} \epsilon^{2} \\
& a_{13}=2 \mu_{0} \frac{q+2 p\left(1+\nu_{0}\right)}{\left(1-\nu_{0}\right)} \epsilon^{2} \quad a_{14}=2 \mu_{0} \frac{q}{1-\nu_{0}} \\
& a_{15}=-2 \mu_{0} \frac{q}{\left(1-\nu_{0}\right)} \epsilon^{2} \quad a_{16}=\mu_{0} \frac{q+4 p\left(1-\nu_{0}\right)}{2\left(1-\nu_{0}\right)} \\
& a_{17}=\mu_{0} \frac{q+2(p-1)}{2\left(1-\nu_{0}\right)} \quad a_{18}=\mu_{0} \frac{-q+2(p-1) \nu_{0}}{2\left(1-\nu_{0}\right)} \\
& a_{19}=-\mu_{0} \frac{q+4 p\left(1-\nu_{0}\right)}{\left(1-\nu_{0}\right)} \epsilon^{2} \quad a_{20}=\mu_{0} \frac{q+8 p \nu_{0}}{4\left(1-\nu_{0}\right)} \\
& a_{21}=\mu_{0} \frac{6+q+2 p+8 p \nu_{0}}{4\left(1-\nu_{0}\right)} \quad a_{22}=-\mu_{0} \frac{q+6(p-1) \nu_{0}}{4\left(1-\nu_{0}\right)} \\
& a_{23}=-\mu_{0} \frac{q+8 p \nu_{0}}{2\left(1-\nu_{0}\right)} \epsilon^{2} \quad a_{24}=-\mu_{0} \frac{q}{1-\nu_{0}} \\
& a_{25}=-\mu_{0} \frac{q+2(p-1) \nu_{0}}{\left(1-\nu_{0}\right)} \quad a_{26}=\mu_{0} \frac{q+2(1-p)}{1-\nu_{0}} \\
& a_{27}=2 \mu_{0} \frac{q}{1-\nu_{0}} \epsilon^{2} \quad a_{28}=-\mu_{0} \frac{q+p\left(3-\nu_{0}\right)}{\left(1-\nu_{0}\right)} \\
& a_{29}=-\mu_{0} \frac{q+2 p\left(1+\nu_{0}\right)}{\left(1-\nu_{0}\right)} \quad a_{30}=\mu_{0} \frac{q}{1-\nu_{0}} \\
& a_{31}=2 \mu_{0} \frac{q+p\left(3-\nu_{0}\right)}{\left(1-\nu_{0}\right)} \epsilon^{2}
\end{aligned}
$$

In the case of a penny-shaped crack, the expansion series of $a_{n}$ for $n=1 . .31$ with respect to the aspect ratio $\epsilon$ can be obtained by replacing $p$ and $q$ by (A.4) in the above expressions. 